\title{
Work Function Changes Induced by Charged Adsorbates: Origin of the Polarity Asymmetry
}

\author{
Paul S. Bagus, ${ }^{1}$ Daniel Käfer, ${ }^{2}$ Gregor Witte, ${ }^{2}$ and Christof Wöll ${ }^{2}$ \\ ${ }^{1}$ Department of Chemistry, University of North Texas, Denton, Texas 76203-5070, USA \\ ${ }^{2}$ Lehrstuhl für Physikalische Chemie I, Ruhr-Universität Bochum, D-44780 Bochum, Germany
}

(Received 16 December 2007; published 26 March 2008)

\begin{abstract}
A theoretical analysis of charged adsorbates on a metal surface reveals a pronounced polarity asymmetry between electropositive and electronegative species, thus reproducing a well known but so far not properly understood experimental fact. For ionic adsorbates on metal surfaces, we analyze the several, often canceling, terms that contribute to the change of the interface dipole and, hence, to workfunction changes, $\Delta \phi$. We demonstrate that for the prototypic case of $\mathrm{I}$ on $\mathrm{Cu}(111)$ the magnitudes and the signs of these terms can be understood on the basis of their physical and chemical origins. An important consequence of their cancellation is that negatively charged adsorbates can lead to a paradoxical $\Delta \phi<0$ rather than the expected $\Delta \phi>0$.
\end{abstract}

The adsorption of atomic or molecular species on metal surfaces induces significant changes of the work function, $\phi[1-4]$. Historically, such changes have been important for lowering the work function to enhance thermal electron emission, e.g., from $W$ filaments [3], and for monitoring adsorption phenomena [5]. Recently, the precise tailoring of the work function of metal surfaces has become an important task with regard to optimizing charge injection at metal-molecule interfaces, in particular, for applications in organic electronics [6]. For strongly electropositive species, e.g., alkali metal atoms, adsorption is accompanied by an electron transfer to the substrate. As first proposed by Langmuir [3], the resulting dipole layer that is directed positive above the surface should result in a strong lowering of the work function [1]. A reasonable approximation for these changes at low coverages is $\Delta \phi \sim$ $-N \Delta \mu$, where $N$ is the adsorbate density and $\Delta \mu$ is the change in the surface dipole induced by a single adsorbate [1]. For relatively low coverages of alkali metals on metal surfaces this model has been successfully used to explain the experimentally observed large lowerings of the work function by $\sim 2-3.5 \mathrm{eV}[1]$.

In principle, the opposite behavior is expected when an electron acceptor is adsorbed on a metal surface with the charge transfer yielding a dipole layer with the opposite orientation, negative above the surface; hence, $\phi$ should increase $[7,8]$. While the expectations derived from the simple Langmuir model have been confirmed many times for electropositive adsorbates, for the opposite case a textbook example, i.e., a case where a simple electronegative adsorbate leads to a work-function increase, is lacking [7,8]. Instead, several cases have been reported [8-10] where an $e$ transfer to the adsorbate is accompanied by a work-function decrease, e.g., I/Ni(100) [9] $(\Delta \phi=$ $-0.8 \mathrm{eV})$ and $\mathrm{I} / \mathrm{Pt}(111)[10](\Delta \phi \sim-0.7 \mathrm{eV})$. Only a few theoretical works have been presented to explain this rather disturbing asymmetry. In earlier work, Pettersson and Bagus [11] analyzed $\mathrm{F}^{-}$and $\mathrm{Cl}^{-}$on $\mathrm{Cu}(100)$ and concluded that polarization of the $\mathrm{Cu}$ surface made an important contribution to $\Delta \phi$. More recently, Michaelides et al. [12] reported that for a charged $\mathrm{N}^{-}$adsorbed on $\mathrm{W}(111)$ the work function is significantly affected by the Pauli exclusion principle. It has been shown earlier by Bagus, Staemmler, and Wöll [13] that this effect explains the surprising lowering of the work function in the case of neutral adsorbates; for rare gas or noble metal systems, see Hückstädt et al. [14] However, N is not a typical electronacceptor and, accordingly, the concept of charge transfer to explain work-function changes is clearly not appropriate for this adsorbate.

In the following, we present a thorough theoretical analysis comparing $\mathrm{Cs}, \mathrm{Xe}$, and I adsorbed on $\mathrm{Cu}(111)$. These adsorbates have similar sizes and hence should be suitable to examine the relation between polarity and work-function change. For the electropositive Cs the experimental findings for $\Delta \phi$ are nicely described by a simple dipole layer model. For the nonpolar Xe, the large adsorption-induced reduction in $\phi$ has recently been shown to result from the Pauli exclusion effect $[13,15]$. However, we shall show that for I, the work function is decreased, in complete contradiction to the expectation of $\Delta \phi>0$ for an electronegative adsorbate. Further, we will show that $\Delta \phi<0$ for I can be directly related to the chemistry of the I-substrate interaction. The theoretical results are validated by our measurements of the workfunction changes for $\mathrm{I}$ on $\mathrm{Cu}(111)$. By using the Helmholtz equation, an interface dipole per I atom, denoted as $\Delta \mu$, of 1.7 $\mathrm{D}$ has been derived from the slope of the measured (using He I UV-photoelectron spectroscopy) $\Delta \phi\left(\theta_{\mathrm{I}}\right)$ curve in the low coverage regime $\left(\theta_{\mathrm{I}}<0.05\right)$.

In our calculations - for details see Refs. $[13,15,16]-$ the unrelaxed $\mathrm{Cu}(111)$ surface is modeled by a three layer, 32 atom $\mathrm{Cu}$ cluster, $\mathrm{Cu}_{32}$, with 19 atoms in the first, 12 in the second, and one in the third layer. This cluster has been used successfully to describe the adsorption of $\mathrm{C}_{6} \mathrm{H}_{6}$ and $\mathrm{C}_{6} \mathrm{H}_{12}$ on $\mathrm{Cu}(111)$ [15]. A Cs, Xe, or I adsorbate, denoted 
$X$, is placed directly above the central first layer atom of $\mathrm{Cu}_{32}$ to form $\mathrm{Cu}_{32} \mathrm{X}$. We chose to place the adsorbates at on-top sites to more easily allow a direct comparison of the various contributions to $\Delta \phi$. The close agreement of our calculated and measured $\Delta \mu$ for $\mathrm{I} / \mathrm{Cu}(111)$ justifies this approach [17]. We then used ab initio methods to obtain wave functions (WFs), energies, and other properties of the clusters. The $1 s$ to $4 d$ core electrons of the I, Xe, and Cs atoms are represented using pseudopotentials with parameters taken from Refs. [18-20], respectively; the basis set for I was augmented with diffuse functions to describe the polarizability [21]. Electron correlation effects were included using second order Moller-Plesset (MP2) perturbation theory [22], because MP2 gives a reliable description of dispersion, or van der Waals, forces [23,24]. The interaction of Cs or I with a metal surface is dominantly an ionic bond, with Cs and I being largely $\mathrm{Cs}^{+}$and $\mathrm{I}^{-}$; however, we will show that a weak dative covalent bond is formed by donation from $\mathrm{I}^{-}$to $\mathrm{Cu}$ and that this bond contributes significantly to the work-function change induced by I. For convenience, the calculations have been carried out for the isoelectronic clusters of $\mathrm{Cu}_{32} \mathrm{I}^{-}, \mathrm{Cu}_{32} \mathrm{Xe}$, and $\mathrm{Cu}_{32} \mathrm{Cs}^{+}$. First, the equilibrium distance, $z_{e}$, of the adsorbate above $\mathrm{Cu}(111)$ was determined from MP2 calculations with the geometry of the copper cluster fixed and only the height of the adsorbate above the surface allowed to vary.

Subsequently, the different contributions to the metaladsorbate interaction and the work-function changes have been obtained by analyzing the properties of the wave functions at the self-consistent field (SCF) level as done successfully in previous work $[13,15,25,26]$. An additional analysis has also been carried out for I kept at the position of Cs. In Table I, we list our calculated $z_{e}$ and $\Delta \mu$ for the three adsorbates. The least strongly bound of the three, Xe, is at the largest distance from $\mathrm{Cu}(111)$ and has the smallest $\Delta \mu$; the calculated $z_{e}$ is only slightly larger than the experimental $z_{e}=3.6 \AA$ [27]. For this case, the sizable reduction of $\Delta \phi$ is dominated by the Pauli exclusion effect [13]. The $\mathrm{Cs}^{+}$adsorbate is substantially, $\sim 0.5 \AA$, closer to $\mathrm{Cu}(111)$ than $\mathrm{Xe}$. This is expected, since $\mathrm{Cs}^{+}$is electrostatically attracted by its "image" charge inside the metal $[7,28,29]$. The large positive $\Delta \mu$ for Cs in Table I is consistent with the large maximum lowerings of $\phi$, by $\sim 3 \mathrm{eV}$, observed for Cs on metal surfaces [1]. The adsorp-

TABLE I. The equilibrium distance, $z_{e}$, and the change in the dipole induced by the adsorbate, $\Delta \mu$, for the $\mathrm{Cu}_{32} \mathrm{Xe}, \mathrm{Cu}_{32} \mathrm{I}^{-}$, and $\mathrm{Cu}_{32} \mathrm{Cs}^{+}$clusters.

\begin{tabular}{lll}
\hline \hline & $z_{e}(\AA)$ & $\Delta \mu$ (Debye) \\
\hline $\mathrm{Xe}$ & 3.86 & +0.23 \\
$\mathrm{Cs}$ & 3.31 & +5.41 \\
$\mathrm{I}$ & 2.74 & +1.50 \\
\hline \hline
\end{tabular}

tion of Cs thus behaves exactly as one would expect following Langmuir and Kingdon [3]. For I/Cu(111), the data in Table I pose surprises: First, the I is drawn in much closer, $\sim 0.5 \AA$, to the surface than is Cs. Since $\mathrm{Cs}^{+}$and $\mathrm{I}^{-}$ are isoelectronic and have comparable sizes [30], one would have expected that the electrostatic interaction between the adsorbed ion and its image would lead to similar $z_{e}$. The second surprise is that the change in the interface dipole is positive, $\Delta \mu=+1.5 \mathrm{D}$, leading to $\Delta \phi<0$ and not negative as expected from a charge transfer (CT) model.

To unravel the origin of this paradoxical behavior for iodine, we have used the constrained space orbital variation (CSOV) methodology [15,31] to decompose the adsorption-induced changes into contributions from different chemical and physical mechanisms. In Table II a decomposition of the interaction energy, $E_{\mathrm{INT}}$, and $\Delta \mu$ is given for $\mathrm{Cu}_{32} \mathrm{Cs}^{+}, \mathrm{Cu}_{32} \mathrm{Xe}$, and $\mathrm{Cu}_{32} \mathrm{I}^{-}$where $E_{\mathrm{INT}}=$ $E\left(\mathrm{Cu}_{32}\right)+E(X)-E\left(\mathrm{Cu}_{32} X\right)$ is $E_{\mathrm{INT}}<0$ for a repulsive interaction and $\Delta \mu=\mu\left(\mathrm{Cu}_{32} X\right)-\mu\left(\mathrm{Cu}_{32}\right)$. Since Hartree-Fock does not include dispersion forces, $\mathrm{Xe} / \mathrm{Cu}$ has $E_{\mathrm{INT}}<0$ while the electrostatic interaction for $\mathrm{Cs} / \mathrm{Cu}$ and $\mathrm{I} / \mathrm{Cu}$ gives $E_{\mathrm{INT}}>0$; see Table II. However, in all three cases, the changes in $E_{\mathrm{INT}}$ for the various CSOV steps show the importance of the chemistry allowed at the step $[13,15]$. In a similar way, the sign and the magnitude of the $\Delta \mu$ at the various CSOV steps give us direct information about the qualitative changes in $\phi$ induced by the adsorption.

The CSOV steps considered [15] are the following: (1) The frozen orbital interaction, denoted Pauli-FO, where the WFs of the separated entities ( $\mathrm{Cu}$ cluster and adsorbate $X)$ are superimposed to form an antisymmetric total WF and only physical changes in the WF (i.e., Pauli exclusion) are taken into account. For the charged adsorbates, we also consider the $\mu$ at an initial CSOV step, denoted point charge (PC)-FO or CSOV step $(0)$, where the adsorbate is represented as a point charge of $\mathrm{PC}=+1\left(\mathrm{Cs}^{+}\right)$or $\mathrm{PC}=$ $-1\left(\mathrm{I}^{-}\right)$. The $\mu$ for a $\mathrm{PC}$ is different from that for an extended ion at the Pauli-FO step because there is no Pauli exclusion for a PC at CSOV step(0). (2) $\mathrm{V}(\mathrm{Cu})$, where the adsorbate orbitals are held fixed but the orbitals of the $\mathrm{Cu}_{32}$ cluster are allowed to vary in the presence of the adsorbate. (3) V(adsorbate), where the substrate orbitals are held fixed after the $\mathrm{V}(\mathrm{Cu})$ variation and the adsorbate orbitals are allowed to vary. (4) The final CSOV step is a full SCF calculation, where no constraints are applied. The changes at this step provide a measure of the nonadditivity of the different CSOV contributions where small changes indicate that the decomposition is fully additive.

For $\mathrm{Xe} / \mathrm{Cu}(111)$, the CSOV decomposition (see Ref. [13]) reveals that the dominant contribution to $\Delta \mu$ comes from the Pauli exclusion; the other CSOV terms contribute only about $30 \%$. In the case of the charged $\mathrm{Cs}^{+}$, the very large dipole seen in steps (0) and (1) is strongly 
TABLE II. A CSOV decomposition of the interaction energy, $E_{\mathrm{INT}}$ in $\mathrm{eV}$, and $\Delta \mu$, in $\mathrm{D}$, for $\mathrm{Xe} / \mathrm{Cu}, \mathrm{Cs} / \mathrm{Cu}$, and I/Cu; see text for the definitions of the CSOV steps and the adsorbate positions, $z(X)$. For the induced dipole, the changes with respect to the bare surface, $\Delta \mu$, and the changes for each CSOV step, $\delta \mu$, are given.

\begin{tabular}{lcccccccc}
\hline \hline \multirow{2}{*}{ CSOV Step } & \multicolumn{2}{c}{$\mathrm{Cu}_{32} \mathrm{Xe} / z(\mathrm{Xe})=3.86 \AA$} & \multicolumn{2}{c}{$\mathrm{Cu}_{32} \mathrm{Cs}^{+} / z(\mathrm{Cs})=3.31 \AA$} & \multicolumn{2}{c}{$\mathrm{Cu}_{32} \mathrm{I}^{-} / z(\mathrm{I})=2.74 \AA$} & \multicolumn{2}{c}{$\mathrm{Cu}_{32} \mathrm{I}^{-} / z(\mathrm{I})=3.31 \AA$} \\
& $E_{\mathrm{INT}}$ & $\Delta \mu(\delta \mu)$ & $E_{\mathrm{INT}}$ & $\Delta \mu(\delta \mu)$ & $E_{\mathrm{INT}}$ & $\Delta \mu(\delta \mu)$ & $E_{\mathrm{INT}}$ & $\Delta \mu(\delta \mu)$ \\
\hline (0) Point charge-FO & $\ldots$ & $\ldots$ & $\ldots$ & +15.9 & $\ldots$ & -13.2 & $\cdots$ & -15.9 \\
(1) Pauli-FO & -0.15 & $+0.32(+0.32)$ & -0.03 & $+16.3(+0.4)$ & -1.78 & $-12.0(+1.1)$ & -0.83 & $-15.4(+0.5)$ \\
(2) V(Cu) & -0.03 & $+0.16(-0.16)$ & +1.16 & $+5.8(-10.5)$ & -0.49 & $-2.8(+9.2)$ & +0.04 & $-6.4(+9.0)$ \\
(3) V(adsorbate) & -0.02 & $+0.23(+0.07)$ & +1.19 & $+5.5(-0.3)$ & +0.41 & $+0.5(+3.4)$ & +0.49 & $-3.8(+2.5)$ \\
(4) Full SCF & -0.02 & $+0.23(-0.00)$ & +1.19 & $+5.4(-0.1)$ & +0.54 & $+1.5(+1.0)$ & +0.53 & $-3.1(+0.7)$ \\
\hline \hline
\end{tabular}

reduced by the polarization of the $\mathrm{Cu}$ substrate (step 2) and the $\Delta \phi$ for an $0.1 \mathrm{ML}$ coverage of $\mathrm{Cs}^{+}$is estimated to be $\sim-3.4 \mathrm{eV}$, consistent with experimental data $[1,32]$. However, this situation changes considerably for I/Cu. In Table II, we show the CSOV analyses for $\mathrm{I} / \mathrm{Cu}$ at $z_{e}=$ $2.74 \AA$ and at $z=3.31 \AA$, which is the $z_{e}$ for $\mathrm{Cs} / \mathrm{Cu}$. For the full SCF variation at the longer distance, $\Delta \mu=$ $-3.1 \mathrm{D}$ is negative, exactly as expected from the CT model. However at the shorter $z_{e}$ for $\mathrm{I} / \mathrm{Cu}(111)$, the work-function change is, unexpectedly, positive, $\Delta \mu=$ +1.5 D. A detailed investigation of the CSOV steps establishes the origin of this apparently paradoxical change of sign in $\Delta \mu$.

We consider first the CSOV for $\mathrm{Cu}_{32} \mathrm{I}^{-}$with $z(\mathrm{I})=$ $2.74 \AA$. The energetic repulsion for the Pauli-FO WF is $-1.8 \mathrm{eV}$, considerably larger than the small repulsion of $-0.03 \mathrm{eV}$ at this $\mathrm{CSOV}$ step for $\mathrm{Cs} / \mathrm{Cu}$. The repulsion between the $\mathrm{Cu}$ surface charge and $\mathrm{I}^{-}$leads to a major reduction of charge in the space between $\mathrm{Cu}$ and $\mathrm{I}$ at the next CSOV step(2) as shown graphically in Fig. 1(a). The $\mathrm{Cu}$ conduction band charge flows mainly into the bulk. The variation of the $\mathrm{I}^{-}$charge at CSOV step (3) leads to substantial changes that are much larger than the changes at this CSOV step for $\mathrm{Cs} / \mathrm{Cu}$. At this step, $\mu$ increases by $3.4 \mathrm{D}$, an increase that is $\sim 10 \times$ larger than that for $\mathrm{Cs} / \mathrm{Cu}$, and $\Delta \mu$ for $\mathrm{I} / \mathrm{Cu}(111)$ becomes positive. The changes in the charge density due to the variation of the $\mathrm{I}^{-}$orbitals are shown in the $\Delta \rho=\rho[\mathrm{V}(\mathrm{I})]-\rho[\mathrm{V}(\mathrm{Cu})]$ in Fig. 1(b). There is a loss of density around I, which is due to a combination of polarization of the spherical $\mathrm{I}^{-}$charge by the positive "image charge" in the surface and by a covalent donation of charge into empty $\mathrm{Cu}$ surface orbitals. The buildup of charge in the region between I and the $\mathrm{Cu}$ surface is a clear demonstration of a covalent bond. The relatively small number of positive contours reveal that the bond is not particularly strong. Further evidence for a donation of charge from $\mathrm{I}^{-}$comes from the projection [33] of the anion orbitals on the WF for $\mathrm{I} / \mathrm{Cu}(111)$

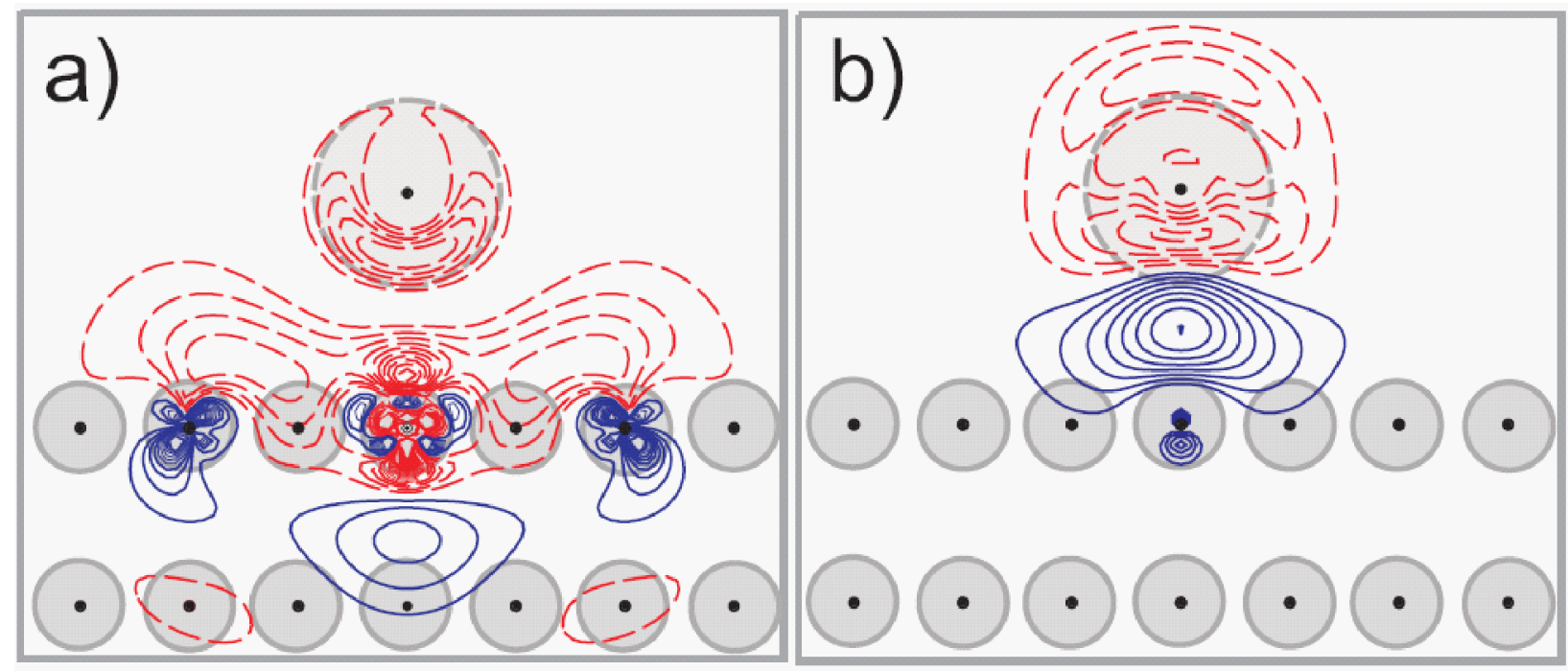

FIG. 1 (color online). Density difference plots for $\mathrm{Cu}_{32} \mathrm{I}$ showing (a) the $\mathrm{Cu}$ polarization and (b) the I polarization and donation. Solid (blue) lines are density increases and dashed (red) lines are density decreases. The plotting plane passes through I and the adsorption site $\mathrm{Cu}$ atom; the positions of the $\mathrm{Cu}$ atoms, projected onto the plotting plane, are shown as shaded circles. (a) $\Delta \rho=\rho[\mathrm{V}(\mathrm{Cu})]-\rho[$ Pauli FO $]$, (b) $\Delta \rho=\rho[\mathrm{V}(\mathrm{I})]-\rho[\mathrm{V}(\mathrm{Cu})]$. 
that gives $5.95 p$ electrons, slightly reduced from the value of 6 for a perfect ionic $\mathrm{I}^{-}$. We have used refinements of the CSOV variations [31] to show that the relative contributions to $\Delta \mu$ from $\mathrm{I}^{-}$polarization and from donation are comparable. For both the shorter $z(\mathrm{I})$ and the longer $z(\mathrm{I})=$ $3.31 \AA$, all of the CSOV variations act to increase $\Delta \mu$ above the point charge value and, at $z(I)=2.74 \AA$, they are sufficiently large so that $\Delta \mu$ changes sign. At the larger $z(\mathrm{I})$, the increases in $\Delta \mu$ are smaller and $\Delta \mu$ remains $\Delta \mu<0$ as expected from the CT model.

In summary, we have shown that, in addition to the dipole layer arising from the charge transfer between an ionic adsorbate and the surface, two other important effects, not symmetric with respect to polarity, must be considered when predicting $\Delta \phi$. First, a purely quantummechanical effect, exchange, or Pauli, exclusion, leads to an adsorbate induced distortion of the metal charge which, for both polarities, tends to reduce the work function. For electropositive adsorbates, like Cs, the effect of the dipole layer is amplified by this effect while for electronegative adsorbates, the two effects act in opposite directions. Second, and more importantly, the anionic adsorbate is much more polarizable, since it has an extra electron, than the cationic adsorbate, which lost an electron. Thus, (weak) covalent chemical bonds are likely to form between the anion and the metal surface. While it was recognized earlier [12] that this covalent bond affects, to some extent, the work function, we show that the main, very important, effect of the covalent bond is a substantial reduction in bond length. At the shorter bond length, the dipole moment created by the negative charge and its positive charge inside the metal is significantly reduced. The combination and partial cancellation of these effects may then even result in a work-function reduction, as demonstrated here for the prototypic case of iodine, thus yielding the opposite of the prediction of the simple electrostatic model [3].

We acknowledge partial support by the NSF (NSF Grant No. CHE06-51083) and the DFG (Grant No. SFB 558) as well as computer support from the National Center for Supercomputing Applications, Urbana-Champaign, IL.

[1] H. P. Bonzel, Surf. Sci. Rep. 8, 43 (1987).

[2] N. D. Lang and W. Kohn, Phys. Rev. B 3, 1215 (1971).

[3] I. Langmuir and K. H. Kingdon, Phys. Rev. 21, 419 (1923).
[4] G. Pacchioni and P. S. Bagus, Surf. Sci. 286, 317 (1993).

[5] J. B. Taylor and I. Langmuir, Phys. Rev. 44, 423 (1933).

[6] J. C. Scott, J. Vac. Sci. Technol. A 21, 521 (2003).

[7] N.W. Ashcroft and N.D. Mermin, Solid State Physics (Holt, Rinehart and Winston, New York, 1976).

[8] M. P. Kiskinova, Surf. Sci. Rep. 8, 359 (1988).

[9] Y. Yang and J. A. Yarmoff, J. Vac. Sci. Technol. A 21, 1317 (2003).

[10] S. K. Jo and J. M. White, Surf. Sci. 261, 111 (1992).

[11] L. G. M. Pettersson and P. S. Bagus, Phys. Rev. Lett. 56, 500 (1986).

[12] A. Michaelides et al., Phys. Rev. Lett. 90, 246103 (2003).

[13] P. S. Bagus, V. Staemmler, and C. Wöll, Phys. Rev. Lett. 89, 096104 (2002).

[14] C. Hückstädt et al., Phys. Rev. B 73, 075409 (2006).

[15] P. S. Bagus, K. Hermann, and C. Wöll, J. Chem. Phys. 123, 184109 (2005).

[16] For I and Cs adsorbates, there is a minor modification for the treatment of $\mathrm{Cu}_{32}$ described in Ref. [13]. Since these adsorbates are closer to the surface than Xe, the adsorption site $\mathrm{Cu}$ atom is treated as an all electron atom to ensure that no artifacts due to the pseudopotential are introduced.

[17] Calculations for I at a threefold site of $\mathrm{Cu}(111)$ show a similar ionicity as at an on-top site although the distance of I above the surface is slightly shorter at the threefold site.

[18] T. Leininger et al., Chem. Phys. Lett. 255, 274 (1996).

[19] W. J. Stevens et al., Can. J. Chem. 70, 612 (1992).

[20] P. J. Hay and W. R. Wadt, J. Chem. Phys. 82, 299 (1985).

[21] P. S. Bagus, G. Pacchioni, and M. R. Philpott, J. Chem. Phys. 90, 4287 (1989).

[22] C. Moller and M. S. Plesset, Phys. Rev. 46, 618 (1934).

[23] X. Wu et al., J. Chem. Phys. 115, 8748 (2001).

[24] M. A. Omary et al., J. Phys. Chem. A 109, 690 (2005).

[25] C. Wöll, K. Weiss, and P. S. Bagus, Chem. Phys. Lett. 332, 553 (2000).

[26] G. Witte et al., Appl. Phys. Lett. 87, 263502 (2005).

[27] T. Seyller et al., Chem. Phys. Lett. 291, 567 (1998).

[28] M. Garcia-Hernandez, P. S. Bagus, and F. Illas, Surf. Sci. 409, 69 (1998).

[29] P. S. Bagus, M. Garcia-Hernandez, and F. Illas, Surf. Sci. 429, 348 (1999).

[30] J. P. Desclaux, At. Data Nucl. Data Tables 12, 311 (1973).

[31] P.S. Bagus, K. Hermann, and C.W. Bauschlicher, Jr., J. Chem. Phys. 80, 4378 (1984).

[32] S. A. Lindgren and L. Wallden, J. Phys. Condens. Matter 2, 5929 (1990).

[33] C. J. Nelin, P. S. Bagus, and M. R. Philpott, J. Chem. Phys. 87, 2170 (1987). 